\title{
Characteristics and Outcomes In Patients With Left- Sided Infective Endocarditis Undergoing Left-Sided Valve Surgery With or Without Concomitant Tricuspid Annuloplasty
}

\section{Zexuan Wu}

Sun Yat-sen University First Affiliated Hospital

Yuanyuan Zhou

Sun Yat-sen University First Affiliated Hospital

Yili Chen

Sun Yat-sen University First Affiliated Hospital

\section{Fangfei Wei}

Sun Yat-sen University First Affiliated Hospital

Zi Ye

St Vincent's College

Xin He

Sun Yat-sen University First Affiliated Hospital

\section{Weihao Liang}

Sun Yat-sen University First Affiliated Hospital

Jingjing Zhao

Sun Yat-sen University First Affiliated Hospital

Ruicong Xue

Sun Yat-sen University First Affiliated Hospital

Yuzhong Wu

Sun Yat-sen University First Affiliated Hospital

Wengen Zhu

Sun Yat-sen University First Affiliated Hospital

\section{Yugang Dong}

Sun Yat-sen University First Affiliated Hospital

Chen Liu ( $\boldsymbol{D}$ liuch75@mail.sysu.edu.cn )

Sun Yat-sen University First Affiliated Hospital

\section{Research Article}

Keywords: Left-sided infective endocarditis, left-side valve surgery, concomitant tricuspid annuloplasty, survival 
Posted Date: April 21st, 2021

DOI: https://doi.org/10.21203/rs.3.rs-406047/v1

License: (c) (1) This work is licensed under a Creative Commons Attribution 4.0 International License. Read Full License 


\section{Abstract}

Background: In patients with left-sided infective endocarditis (LSIE) undergoing left-sided valve surgery (LVS), the effects of concomitant tricuspid annuloplasty (TA) on clinical features and prognosis remain unknown.

Methods: This is a single-center retrospective study conducted in a tertiary hospital in China. A total of 207 consecutive patients $\geq 18$ years with a definite LSIE diagnosis who underwent LVS between 2008 and 2017 were included. Patients were divided into two groups: LVS alone $(n=157)$ and LVS+TA group $(n=50)$, to identify differences between the clinical features, echocardiogdraphic parameters and outcomes.

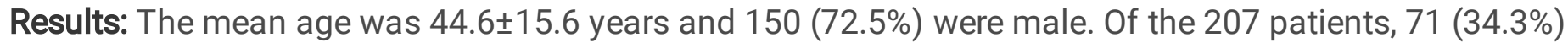
patients had aortic valve involved alone, 115 (55.6\%) had mitral valve involved alone and 21 (10.1\%) had both valved involved. The average hospital stays were $38 \pm 16$ days and the median follow-up duration was 34.4 (IQR 19.8-56.3) months. Demographic and baseline characteristics were comparable between the two groups, except that the renal function in LVS alone group was better than LVS +TA group (eGFR $97.2 \pm 28.8 \mathrm{vs}$. 87.6 $\pm 30.7, P=0.046)$. Significant reductions in left and right atrial diameter, left ventricular diameter, mitral and tricuspid regurgitation (TR) degree, and pulmonary arterial systolic pressure were reported in both groups, of which the differences were more prominent in LVS+ TA group than LVS alone group $(P<0.05)$. The rate of postoperative complications was higher in LVS+ TA group than that in LVS group (44.0\% vs. $23.6 \%, P=0.005)$. However, the in-hospital mortality and long-term mortality was similar in both groups. After multi-factor adjustment, concomitant TA was not significantly associated with in-hospital and long-term mortality.

Conclusions: Concomitant TA at the time of LVS significantly improved cardiac diameter but increased postoperative complications. It might not be associated with improved survival in LSIE patients.

\section{Introduction}

Despite the improvements in medical and surgical interventions, infective endocarditis remains a potentially lethal disease with the mortality ranging from 20-30\% [1-4]. Early surgery significantly decreases the allcause mortality and the incidence of embolic events in patients with left-sided infective endocarditis (LSIE) compared with conservative management, and it is recommended for patients meeting specific indications such as severe valve dysfunction [5-7]. As a prevalent condition, tricuspid regurgitation (TR) has attracted more and more discussions. Over $80 \%$ of tricuspid regurgitation is attributed to secondary (functional) causes including tricuspid annuli and right ventricle dilation, while primary causes account for less than $20 \%$ of TR. Functional TR is often secondary to left-sided heart disease, predominantly mitral valve disease [8-10]. For patients undergoing mitral-valve surgery, the rate of functional TR is up to $50 \%$ [11]. Recent study has revealed that increasing TR severity is correlated with worse prognosis regardless of left ventricular function or pulmonary hypertension, and severe TR may not predictably improve after left-sided valve surgery [6]. Nevertheless, concomitant tricuspid valve repair during left-sided valve surgery is beneficial from reducing cardiac-related mortality [12]. Consequently, the American valvular heart disease guideline recommends concomitant tricuspid annuloplasty (TA) for patients with severe TR undergoing left-sided valve surgery (LVS) [13]. However, there is a paucity of evidence available to define the benefit of concomitant TV surgery in paitents with LSIE who need LVS. Most studies for the surgical management of functional TR are based on 
rheumatic or degenerative valvular disease, while IE accounts for only $16 \%$ of the etiology of left-sided heart disease [14]. Unlike the chronic onset and presence of the latent phase in rheumatic or degenerative valvular disease, IE commonly presents with more acute onset and more aggressive progression due to different underlying pathophysiology. Thus, the previous evidence from rheumatic or degenerative valvular disease can hardly extended to LSIE patients. In addition, most recommendations are categorized as Level of Evidence C, indicating the lack of high-quality trial or observational data. Therefore, our study aims to evaluate the effect of concomitant TA on clinical features and prognosis at the time of LVS among LSIE patients.

\section{Methods}

\section{Study Population}

This is a single-center retrospective observational study conducted in a tertiary referral hospital, the First Affiliated Hospital of Sun Yat-sen University. From January $1^{\text {st }}$ of 2008 to September $30^{\text {th }}$ of 2017 , a total of 480 consecutive patients with clinical suspicion of IE were screened. We excluded patients who were younger than 18 years old $(n=32)$, without definite IE $(n=34)$ according to the modified Duke Criteria were included in the study [15], and without comprehensive echocardiographic evidence of endocarditis $(n=32)$. Patients with evidence of right-sided endocarditis $(n=37)$, cardiac implantable electronic devices (pacemakers and implantable cardioverter-defibrillators) $(n=4)$, both-sided IE $(n=11)$, and congenital heart disease-related IE $(n=21)$ were also excluded, given these subtypes have completely distinct clinical, microbiological, and prognostic characteristics from LSIE. We also excluded 102 patients who didn't undergo IE surgery. Finally, a total of 207 patients $\geq 18$ years old with a definite LSIE diagnosis undergoing LVS were included in the study

(Figure 1). Patients were further divided into two groups depending on the type of definitive interventions they received: LVS group ( $n=157)$, and LVS+TA group $(n=50)$. The LVS group conducted left-sided valve surgery alone, while the LVS+ TA group underwent concomitant TA with LVS.

\section{Definition of terms}

The estimated glomerular filtration ratio (eGFR) was calculated using the 2009 CKD-EPI (Chronic Kidney Disease Epidemiology Collaboration) creatinine $\left(\mathrm{eGFR}_{\mathrm{cr}}\right)$ equation [16]. The presence of heart failure was determined by symptoms and signs elicited from clinical assessment, laboratory investigations, and radiographic findings at baseline preoperatively $[17,18]$. Systemic embolisms were defined as acute onset of clinical symptoms or signs indicating a lodging embolus involving the brain, spleen, kidneys, or lung, consistent with radiographic evidence [19]. Vascular phenomena, such as cutaneous microinfarctions and metastatic abscesses, were not classified under systemic embolisms. Neurological complications included meningoencephalopathies, ischemic complications, cerebral hemorrhage, and intracranial abscess [20]. According to previous studies and guidelines, paravalvular complications were defined as intracardiac abscesses or fistulas based on transthoracic or transesophageal echocardiograms [21, 22]. Postoperative complications included major bleeding, neurological complications, cardiac complications, infection, and thoracic effusion or pneumothorax and poor wound healing. Major bleeding was defined as: 1) intracranial bleeding; 2) overt bleeding resulting in a decrease in hemoglobin $\geq 20 \mathrm{~g} / \mathrm{L}$ or requiring blood transfusion; or 3 ) bleeding into a confined space, such as pericardial cavity or paraspinal space, which indicates severe morbidity. The definition of neurological complications was above-mentioned. Cardiac complications 
included heart failure, arrhythmia, massive pericardial effusion, and low cardiac output syndrome. The study outcome for this analysis was in-hospital and long-term all-cause mortality. Since the high in-hospital mortality rate, the long-term all-cause mortality was calculated from both index admission and from discharge respectively.

\section{Data collection and measurement of echocardiographic parameters}

Baseline data were collected from the electronic medical record system, including the patients' demographic characteristics, previous history of underlying diseases, clinical presentations, laboratory test results on admission, transthoracic/transesophageal echocardiographic data, complications and microscopic studies of associated pathogens. The clinical severities were assessed by the Pitt bacteremia scores (PBS) ranging from 0 to 14 points with the higher score indicating the severer status [23].

Transthoracic echocardiograms (TTE) were performed at baseline preoperatively and postoperatively. The median duration of postoperative TTE performed were 8 (inter-quartile range, IQR: 7-13) days post operation. Left ventricular ejection fraction (LVEF) was measured by Simpson's method. In accordance of the recommendations from American Society of Echocardiography, the severity of tricuspid regurgitation was assessed using color Doppler flow images in TTE and graded qualitatively as mild (1+), moderate (2+), or severe (3+ or $4+)$ following the jet area-central jets of $<5,5-10$, or $>10 \mathrm{~cm}^{2}$ respectively [24]. The pulmonary artery systolic pressure was calculated as the addition of estimated right atrial pressure (ranging from 5 to $10 \mathrm{mmHg}$ given the varied size of inferior vena cava) and the systolic right atrial-ventricular pressure gradient $(P G / \Delta P)$, which was calculated by the modified Bernoulli equation: $\Delta P=4 \times v^{2}(v$ : the maximal velocity of the TR jet area) [25].

\section{Surgery procedures}

All operations were performed under conventional cardiopulmonary bypass, mild or moderate hypothermia through median sternotomy. Mitral or aortic valve surgeries were performed before examining the tricuspid valve. For patients who underwent concomitant TA, either De Vega annuloplasty or ring annuloplasty were used according to the surgeon's comprehensive evaluations following the latest guidelines' recommendations at that time [26-28].

\section{Statistical Analysis}

Continuous normally distributed variables were described as mean value with standard deviation (SD) or median (IQR) when appropriate. For quantitative variables, the groups were compared by a two-tailed Student's t-test or Mann-Whitney U-test when necessary. Categorical variables were expressed as number of event and a percentage, and they were examined by the $\chi 2$ test or Fisher's exact test when appropriate. Categorical echocardiographic variables change between pre- and post-operation were analyzed using McNemar's statistical test.

Multivariable logistic regression models were applied to determine whether concomitant TA were associated with in-hospital mortality postoperation after adjusting for confounding factors. Multivariable cox regression models were used to explore the association of concomitant TA with long-term survival adjusted for 
predefined covariates. The survival time was calculated from death or the last time of follow up to hospital discharge. Since the high rate of in-hospital mortality, the survival time between death or last time of follow up to hospital admission were also discussed.

All hypothesis tests were two-sided, and a $\mathrm{P}$ value $<0.05$ was considered as statistically significant. Statistical analysis was performed with SPSS software V22.0 (SPSS Inc., Chicago, IL, USA).

\section{Results}

\section{Baseline Characteristics of Patients}

The baseline characteristics of the overall study population were demonstrated in Table 1. The mean age was $44.6 \pm 15.6$ years and $150(72.5 \%)$ were male. $97.1 \%$ of the cohort were native IE patients, while only $2.9 \%$ were prosthetic IE patients. 71 (34.3\%) of patients had aortic valve involved alone, $115(55.6 \%)$ had mitral valve involved while $21(10.1 \%)$ had both aortic and mitral valves involved. A total of $157(75.8 \%)$ patients underwent LVS alone while 50 (24.2\%) underwent concomitant TA. Of the 50 patients underwent concomitant TA, 18 (36.0\%) received De Vega annuloplasty while 32 (54.0\%) received ring annuloplasty. 
Table 1

Baseline Characteristics of Patients with LSIE

\begin{tabular}{|c|c|c|c|c|}
\hline Variables & $\begin{array}{l}\text { All } \\
(n=207)\end{array}$ & $\begin{array}{l}\text { LVS } \\
(n=157)\end{array}$ & $\begin{array}{l}\text { LVS + TA } \\
(n=50)\end{array}$ & $P$ value \\
\hline Age (yrs) & $44.6 \pm 15.6$ & $44.0 \pm 15.7$ & $46.2 \pm 15.1$ & 0.388 \\
\hline Male, n (\%) & $150(72.5)$ & $110(71.0)$ & $40(80.0)$ & 0.171 \\
\hline Atrial fibrillation, n (\%) & $18(8.7)$ & $10(6.4)$ & $8(16.0)$ & 0.035 \\
\hline Hypertension, n (\%) & $37(17.9)$ & $29(18.5)$ & $8(16.0)$ & 0.691 \\
\hline Diabetes mellitus, n (\%) & $10(4.8)$ & $9(5.7)$ & $1(2.0)$ & 0.284 \\
\hline Coronary artery disease, $\mathrm{n}(\%)$ & $16(7.7)$ & $12(7.6)$ & $4(8.0)$ & 0.934 \\
\hline Rheumatic heart disease, $\mathrm{n}(\%)$ & $31(15.0)$ & $22(14.0)$ & $9(18.0)$ & 0.491 \\
\hline COPD, n (\%) & $3(1.4)$ & $2(1.3)$ & $1(2.0)$ & 0.708 \\
\hline Chronic kidney disease, n (\%) & $18(8.7)$ & $16(10.2)$ & $2(4.0)$ & 0.176 \\
\hline NYHA class, n (\%) & & & & 0.712 \\
\hline Class I & $14(6.8)$ & $12(7.6)$ & $2(4.0)$ & \\
\hline Class II & $68(32.9)$ & $52(33.1)$ & $16(32.0)$ & \\
\hline Class III & $109(52.7)$ & $80(51.0)$ & $29(58.0)$ & \\
\hline Class IV & $16(7.7)$ & $13(8.3)$ & $3(6.0)$ & \\
\hline Serum creatine (mg/dl) & $0.8(0.7-1.0)$ & $0.8(0.7-1.0)$ & $0.9(0.8-1.3)$ & 0.019 \\
\hline eGFR (ml/min/1.73m²) & $94.9 \pm 29.5$ & $97.2 \pm 28.8$ & $87.6 \pm 30.7$ & 0.046 \\
\hline Types of IE, n (\%) & & & & 0.133 \\
\hline Left-sided native IE & $201(97.1)$ & $154(98.1)$ & $47(94.0)$ & \\
\hline Left-sided prosthetic IE & $6(2.9)$ & $3(1.9)$ & $3(6.0)$ & \\
\hline Valve involved, n (\%) & & & & 0.728 \\
\hline Aortic & $71(34.3)$ & $55(35.0)$ & $16(32.0)$ & \\
\hline Mitral & $115(55.6)$ & $85(54.1)$ & $30(60.0)$ & \\
\hline Aortic and mitral & $21(10.1)$ & $17(10.8)$ & $4(8.0)$ & \\
\hline \multicolumn{5}{|c|}{$\begin{array}{l}\text { IE, infective endocarditis; LSIE, left-sided infective endocarditis; LVS, left-sided valve surgery only; TA, } \\
\text { tricuspid annuloplasty; LVS + TA, left-sided valve surgery with concomitant tricuspid annuloplasty; COPD, } \\
\text { chronic obstructive pulmonary disease; NYHA, New York heart association; eGFR, estimated glomerular } \\
\text { filtration rate derived from serum creatinine according to CKD-EPI. }\end{array}$} \\
\hline \multicolumn{5}{|c|}{$\begin{array}{l}\text { *Of the } 32 \text { patients with negative cultures, } 12 \text { of } 24(54.5 \%) \text { in the LVS group and } 3 \text { of } 10(30.0 \%) \text { in the } \\
\text { LVS + TA group had a history of antibiotic use. }\end{array}$} \\
\hline
\end{tabular}




\begin{tabular}{|c|c|c|c|c|}
\hline Variables & $\begin{array}{l}\text { All } \\
(n=207)\end{array}$ & $\begin{array}{l}\text { LVS } \\
(n=157)\end{array}$ & $\begin{array}{l}\text { LVS + TA } \\
(n=50)\end{array}$ & $P$ value \\
\hline Causative microorganism, $\mathrm{n}(\%)$ & & & & 0.195 \\
\hline Streptococcus & $61(29.5)$ & $52(33.1)$ & $9(18.0)$ & \\
\hline Staphylococcus aureus & $10(4.8)$ & $8(5.1)$ & $2(4.0)$ & \\
\hline Others & $104(50.2)$ & $75(47.8)$ & $29(58.0)$ & \\
\hline Negative culture* & $32(15.5)$ & $22(14.0)$ & $10(20.0)$ & \\
\hline Pitt score, IQR & $1.0(0-2.0)$ & $1.0(0-3.0)$ & $1.0(0-2.0)$ & 0.134 \\
\hline \multicolumn{5}{|c|}{$\begin{array}{l}\text { IE, infective endocarditis; LSIE, left-sided infective endocarditis; LVS, left-sided valve surgery only; TA, } \\
\text { tricuspid annuloplasty; LVS + TA, left-sided valve surgery with concomitant tricuspid annuloplasty; COPD, } \\
\text { chronic obstructive pulmonary disease; NYHA, New York heart association; eGFR, estimated glomerular } \\
\text { filtration rate derived from serum creatinine according to CKD-EPI. }\end{array}$} \\
\hline \multicolumn{5}{|c|}{$\begin{array}{l}\text { *Of the } 32 \text { patients with negative cultures, } 12 \text { of } 24(54.5 \%) \text { in the LVS group and } 3 \text { of } 10(30.0 \%) \text { in the } \\
\text { LVS + TA group had a history of antibiotic use. }\end{array}$} \\
\hline
\end{tabular}

Demographic and baseline characteristics were comparable between the two groups, except that patients undergoing LVS with concomitant TA had significantly higher rate of atrial fibrillation $(16.0 \%$ vs. $6.4 \%, P=$ 0.035 ) and worse renal function than those without TA (eGFR $87.6 \pm 30.7$ vs $97.2 \pm 28.8, P=0.046$ ). The Pitt bacteremia scores were comparable between the two groups $(P=0.134)$.

\section{Echocardiographic characteristics}

A comparison of echocardiographic characteristics among patients undergoing left-sided surgery with or without concomitant TA was presented in Table 2. At baseline, the LVS + TA group had greater left atrial diameter (LA), right atrial diameter (RA), right ventricular diameter (RV), more severe mitral regurgitation (MR) and TR, higher PASP as well as larger vegetations compared with LVS group $(P<0.05)$. 
Table 2

Echocardiographic results pre-operation and post-operation in patients undergoing left-sided valve surgery with or without concomitant tricuspid annuloplasty (TA).

\begin{tabular}{|c|c|c|c|c|c|c|c|c|}
\hline \multirow[t]{2}{*}{ Variables } & \multirow{2}{*}{$\begin{array}{l}\text { All } \\
(n= \\
207)\end{array}$} & \multicolumn{3}{|c|}{ LVS ( $n=157)$} & \multicolumn{3}{|c|}{ LVS + TA $(n=50)$} & \multirow{2}{*}{$\begin{array}{l}P \\
\text { valuet }\end{array}$} \\
\hline & & $\begin{array}{l}\text { Pre- } \\
\text { operation }\end{array}$ & $\begin{array}{l}\text { Post- } \\
\text { operation }\end{array}$ & $\Delta \pm S D$ & $\begin{array}{l}\text { Pre- } \\
\text { operation }\end{array}$ & $\begin{array}{l}\text { Post- } \\
\text { operation }\end{array}$ & $\Delta \pm S D$ & \\
\hline $\mathrm{LA}(\mathrm{mm})$ & $\begin{array}{l}42.7 \\
\pm 9.0\end{array}$ & $40.7 \pm 8.0$ & $\begin{array}{l}34.3 \pm \\
6.6^{\#}\end{array}$ & $\begin{array}{l}-6.2 \pm \\
7.1\end{array}$ & $\begin{array}{l}47.8 \pm \\
9.0 \dagger\end{array}$ & $\begin{array}{l}39.4 \pm \\
7.6^{\#}\end{array}$ & $\begin{array}{l}-8.8 \pm \\
8.2\end{array}$ & 0.040 \\
\hline RA_LAD (mm) & $\begin{array}{l}46.3 \\
\pm 7.7\end{array}$ & $44.8 \pm 7.2$ & $\begin{array}{l}41.9 \pm \\
7.3^{\#}\end{array}$ & $\begin{array}{l}-2.9 \pm \\
5.6\end{array}$ & $\begin{array}{l}51.1 \pm \\
7.4 \dagger\end{array}$ & $\begin{array}{l}41.7 \pm \\
7.2^{\#}\end{array}$ & $\begin{array}{l}-8.6 \pm \\
8.6\end{array}$ & $\begin{array}{l}<.001 \\
0.001\end{array}$ \\
\hline RA_SAD (mm) & $\begin{array}{l}35.8 \\
\pm 6.6\end{array}$ & $34.7 \pm 6.2$ & $\begin{array}{l}35.7 \pm \\
5.8\end{array}$ & $\begin{array}{l}+0.8 \\
\pm 4.9\end{array}$ & $\begin{array}{l}39.1 \pm \\
6.9+\end{array}$ & $\begin{array}{l}36.8 \pm \\
5.9\end{array}$ & $\begin{array}{l}-2.3 \pm \\
7.1\end{array}$ & 0.001 \\
\hline LVEDD (mm) & $\begin{array}{l}57.6 \\
\pm \\
10.2\end{array}$ & $57.5 \pm 9.0$ & $\begin{array}{l}45.4 \pm \\
7.7^{\#}\end{array}$ & $\begin{array}{l}-11.8 \\
\pm 7.5\end{array}$ & $\begin{array}{l}58.1 \pm \\
13.5\end{array}$ & $\begin{array}{l}48.0 \pm \\
10.9^{\#}\end{array}$ & $\begin{array}{l}-10.1 \\
\pm 14.9\end{array}$ & 0.324 \\
\hline LVESD (mm) & $\begin{array}{l}36.1 \\
\pm 7.4\end{array}$ & $35.7 \pm 6.9$ & $\begin{array}{l}31.7 \pm \\
6.6^{\#}\end{array}$ & $\begin{array}{l}-3.8 \pm \\
5.6\end{array}$ & $\begin{array}{l}37.2 \pm \\
8.6\end{array}$ & $\begin{array}{l}34.5 \pm \\
8.4^{\#}\end{array}$ & $\begin{array}{l}-3.3 \pm \\
7.2\end{array}$ & 0.701 \\
\hline $\mathrm{RV}(\mathrm{mm})$ & $\begin{array}{l}20.9 \\
\pm 4.4\end{array}$ & $20.4 \pm 4.1$ & $\begin{array}{l}19.7 \pm \\
3.4\end{array}$ & $\begin{array}{l}-0.6 \pm \\
4.7\end{array}$ & $\begin{array}{l}22.5 \pm \\
5.2 \dagger\end{array}$ & $\begin{array}{l}22.1 \pm \\
3.7\end{array}$ & $\begin{array}{l}-0.4 \pm \\
5.6\end{array}$ & 0.772 \\
\hline IVS (mm) & $\begin{array}{l}10.3 \\
\pm 2.3\end{array}$ & $10.2 \pm 2.1$ & $\begin{array}{l}11.0 \pm \\
2.8^{\#}\end{array}$ & $\begin{array}{l}+0.8 \\
\pm 2.7\end{array}$ & $\begin{array}{l}10.5 \pm \\
3.1\end{array}$ & $\begin{array}{l}12.2 \pm \\
5.7^{\#}\end{array}$ & $\begin{array}{l}+1.6 \\
\pm 5.4\end{array}$ & 0.188 \\
\hline LVPW (mm) & $\begin{array}{l}9.4 \pm \\
1.9\end{array}$ & $9.3 \pm 1.7$ & $\begin{array}{l}10.1 \pm \\
2.5^{\#}\end{array}$ & $\begin{array}{l}+0.8 \\
\pm 2.7\end{array}$ & $9.5 \pm 2.3$ & $\begin{array}{l}11.0 \pm \\
4.9\end{array}$ & $\begin{array}{l}+1.3 \\
\pm 4.6\end{array}$ & 0.369 \\
\hline EF (\%) & $\begin{array}{l}66.7 \\
\pm 8.5\end{array}$ & $67.0 \pm 8.7$ & $\begin{array}{l}60.0 \pm \\
9.2^{\#}\end{array}$ & $\begin{array}{l}-7.6 \pm \\
10.4\end{array}$ & $\begin{array}{l}66.2 \pm \\
8.0\end{array}$ & $\begin{array}{l}58.5 \pm \\
12.0^{\#}\end{array}$ & $\begin{array}{l}-7.4 \pm \\
12.2\end{array}$ & 0.937 \\
\hline E/A ratio & $\begin{array}{l}1.4 \pm \\
0.8\end{array}$ & $1.4 \pm 0.7$ & $1.0 \pm 0.5^{\#}$ & $\begin{array}{l}-0.5 \pm \\
1.0\end{array}$ & $1.7 \pm 0.9$ & $1.2 \pm 0.4^{\#}$ & $\begin{array}{l}-1.0 \pm \\
2.7\end{array}$ & 0.602 \\
\hline$E / E^{\prime}$ ratio & $\begin{array}{l}12.4 \\
\pm 5.5\end{array}$ & $11.5 \pm 5.7$ & $\begin{array}{l}14.0 \pm \\
6.1\end{array}$ & $\begin{array}{l}+3.4 \\
\pm 7.3\end{array}$ & $\begin{array}{l}14.3 \pm \\
4.6\end{array}$ & $\begin{array}{l}14.0 \pm \\
1.1\end{array}$ & $\begin{array}{l}+3.7 \\
\pm 5.1\end{array}$ & 0.963 \\
\hline
\end{tabular}

LVS, left-sided valve surgery only; TA, tricuspid annuloplasty; LVS + TA, left-sided valve surgery with concomitant tricuspid annuloplasty; EF, ejection fraction; LA, left atrial; RA_LAD, right atrial long axis diameter; RA_SAD, right atrial short axis diameter; LVEDD, left ventricular end-diastolic diameter; LVESD, left ventricular end-systolic diameter; RV, right ventricular; IVS, interventricular septum; LVPW, left ventricular posterior wall; PASP, pulmonary artery systolic pressure; TR, tricuspid regurgitation; MR, mitral regurgitation; PR, pulmonary regurgitation; AR, aortic regurgitation; CTR, chordae tendineae rupture.

* $\mathrm{P}<0.05$ for the echocardiographic changes $(\Delta)$ between LVS and LVS + TA group.

† $\mathrm{P}<0.05$ versus LVS group preoperation.

\# $\mathrm{P}<0.05$ postoperation versus preoperation. 


\begin{tabular}{|c|c|c|c|c|c|c|c|c|}
\hline \multirow[t]{2}{*}{ Variables } & \multirow{2}{*}{$\begin{array}{l}\text { All } \\
(n= \\
207)\end{array}$} & \multicolumn{3}{|c|}{ LVS $(n=157)$} & \multicolumn{3}{|c|}{ LVS + TA $(n=50)$} & \multirow{2}{*}{$\begin{array}{l}P \\
\text { value* }\end{array}$} \\
\hline & & $\begin{array}{l}\text { Pre- } \\
\text { operation }\end{array}$ & $\begin{array}{l}\text { Post- } \\
\text { operation }\end{array}$ & $\Delta \pm S D$ & $\begin{array}{l}\text { Pre- } \\
\text { operation }\end{array}$ & $\begin{array}{l}\text { Post- } \\
\text { operation }\end{array}$ & $\Delta \pm S D$ & \\
\hline $\begin{array}{l}\text { Diastolic } \\
\text { dysfunction, } \mathrm{n} \\
(\%)\end{array}$ & $\begin{array}{l}68 \\
(32.9)\end{array}$ & $51(32.5)$ & $30(19.1)$ & $-10.8 \%$ & $17(34.0)$ & $9(18.0)$ & $-16.0 \%$ & \\
\hline PASP $(\mathrm{mmHg})$ & $\begin{array}{l}44.9 \\
\pm \\
15.2\end{array}$ & $\begin{array}{l}41.9 \pm \\
13.9\end{array}$ & $\begin{array}{l}30.6 \pm \\
8.3^{\#}\end{array}$ & $\begin{array}{l}-9.2 \pm \\
13.6\end{array}$ & $\begin{array}{l}51.4 \pm \\
16.3 \dagger\end{array}$ & $\begin{array}{l}29.6 \pm \\
6.7^{\#}\end{array}$ & $\begin{array}{l}-24.1 \\
\pm 16.8\end{array}$ & $\begin{array}{l}< \\
0.001\end{array}$ \\
\hline TR & $\begin{array}{l}1.18 \\
\pm \\
0.58\end{array}$ & $\begin{array}{l}1.04 \pm \\
0.48\end{array}$ & $\begin{array}{l}0.94 \pm \\
0.33^{\#}\end{array}$ & $\begin{array}{l}-0.10 \\
\pm 0.54\end{array}$ & $\begin{array}{l}1.64 \pm \\
0.63 \dagger\end{array}$ & $\begin{array}{l}0.94 \pm \\
0.24^{\#}\end{array}$ & $\begin{array}{l}-0.70 \\
\pm 0.68\end{array}$ & $<$ \\
\hline MR & $\begin{array}{l}2.18 \\
\pm \\
0.90\end{array}$ & $\begin{array}{l}2.11 \pm \\
0.92\end{array}$ & $\begin{array}{l}0.58 \pm \\
0.53^{\#}\end{array}$ & $\begin{array}{l}-1.54 \\
\pm 1.02\end{array}$ & $\begin{array}{l}2.40 \pm \\
0.78 \dagger\end{array}$ & $\begin{array}{l}0.50 \pm \\
0.51^{\#}\end{array}$ & $\begin{array}{l}-1.90 \\
\pm 1.04\end{array}$ & 0.030 \\
\hline PR & $\begin{array}{l}0.86 \\
\pm \\
0.48\end{array}$ & $\begin{array}{l}0.87 \pm \\
0.45\end{array}$ & $\begin{array}{l}0.80 \pm \\
0.40\end{array}$ & $\begin{array}{l}-0.06 \\
\pm 0.51\end{array}$ & $\begin{array}{l}0.86 \pm \\
0.57\end{array}$ & $\begin{array}{l}0.88 \pm \\
0.33\end{array}$ & $\begin{array}{l}-0.02 \\
\pm 0.59\end{array}$ & 0335 \\
\hline AR & $\begin{array}{l}1.29 \\
\pm \\
1.24\end{array}$ & $\begin{array}{l}1.30 \pm \\
1.25\end{array}$ & $\begin{array}{l}0.42 \pm \\
0.50^{\#}\end{array}$ & $\begin{array}{l}-0.88 \\
\pm 1.05\end{array}$ & $\begin{array}{l}1.28 \pm \\
1.25\end{array}$ & $\begin{array}{l}0.38 \pm \\
0.64^{\#}\end{array}$ & $\begin{array}{l}-0.90 \\
\pm 1.16\end{array}$ & 0.905 \\
\hline $\begin{array}{l}\text { TR< } \\
\text { moderate, } n \\
(\%)\end{array}$ & $\begin{array}{l}158 \\
(76.3)\end{array}$ & $136(86.6)$ & $\begin{array}{l}137 \\
(87.3)\end{array}$ & $+0.7 \%$ & $\begin{array}{l}22(44.0) \\
+\end{array}$ & $50(100)$ & $\begin{array}{l}+ \\
56.0 \%\end{array}$ & - \\
\hline $\begin{array}{l}\text { MR< } \\
\text { moderate, } n \\
(\%)\end{array}$ & $\begin{array}{l}53 \\
(25.6)\end{array}$ & $44(28.0)$ & $\begin{array}{l}141 \\
(89.8)\end{array}$ & $\begin{array}{l}+ \\
61.8 \%\end{array}$ & $9(18.0)$ & $50(100)$ & $\stackrel{+}{82.0 \%}$ & - \\
\hline $\begin{array}{l}\mathrm{PR}< \\
\text { moderate, } \mathrm{n} \\
(\%)\end{array}$ & $\begin{array}{l}197 \\
(95.2)\end{array}$ & $\begin{array}{l}150 \\
(95.5)\end{array}$ & $\begin{array}{l}141 \\
(89.8)\end{array}$ & $-0.7 \%$ & $47(94.0)$ & $50(100)$ & $+6.0 \%$ & - \\
\hline $\begin{array}{l}\text { AR< } \\
\text { moderate, n } \\
(\%)\end{array}$ & $\begin{array}{l}120 \\
(58.0)\end{array}$ & $89(56.7)$ & $\begin{array}{l}142 \\
(90.4)\end{array}$ & $\stackrel{+}{33.7 \%}$ & $31(62.0)$ & $48(96.0)$ & $\stackrel{+}{34.0 \%}$ & - \\
\hline
\end{tabular}

LVS, left-sided valve surgery only; TA, tricuspid annuloplasty; LVS + TA, left-sided valve surgery with concomitant tricuspid annuloplasty; EF, ejection fraction; LA, left atrial; RA_LAD, right atrial long axis diameter; RA_SAD, right atrial short axis diameter; LVEDD, left ventricular end-diastolic diameter; LVESD, left ventricular end-systolic diameter; RV, right ventricular; IVS, interventricular septum; LVPW, left ventricular posterior wall; PASP, pulmonary artery systolic pressure; TR, tricuspid regurgitation; MR, mitral regurgitation; $\mathrm{PR}$, pulmonary regurgitation; $\mathrm{AR}$, aortic regurgitation; CTR, chordae tendineae rupture.

* $\mathrm{P}<0.05$ for the echocardiographic changes $(\Delta)$ between LVS and LVS + TA group.

† $\mathrm{P}<0.05$ versus LVS group preoperation.

$\# \mathrm{P}<0.05$ postoperation versus preoperation. 


\begin{tabular}{|c|c|c|c|c|c|c|c|c|}
\hline \multirow[t]{2}{*}{ Variables } & \multirow{2}{*}{$\begin{array}{l}\text { All } \\
(n= \\
207)\end{array}$} & \multicolumn{3}{|c|}{ LVS $(n=157)$} & \multicolumn{3}{|c|}{ LVS + TA $(n=50)$} & \multirow{2}{*}{$\begin{array}{l}P \\
\text { valuet }\end{array}$} \\
\hline & & $\begin{array}{l}\text { Pre- } \\
\text { operation }\end{array}$ & $\begin{array}{l}\text { Post- } \\
\text { operation }\end{array}$ & $\Delta \pm \mathrm{SD}$ & $\begin{array}{l}\text { Pre- } \\
\text { operation }\end{array}$ & $\begin{array}{l}\text { Post- } \\
\text { operation }\end{array}$ & $\Delta \pm \mathrm{SD}$ & \\
\hline $\begin{array}{l}\text { Vegetation } \\
\text { size }(\mathrm{mm})\end{array}$ & $\begin{array}{l}12.5 \\
\pm 6.1\end{array}$ & $11.7 \pm 5.6$ & - & - & $\begin{array}{l}15.0 \pm \\
6.9 \dagger\end{array}$ & - & - & - \\
\hline $\begin{array}{l}\text { Vegetation > } \\
10 \mathrm{~mm}\end{array}$ & $\begin{array}{l}88 \\
(42.5)\end{array}$ & $60(38.2)$ & - & - & $28(56.0)$ & - & - & - \\
\hline $\begin{array}{l}\text { Vegetation > } \\
15 \mathrm{~mm}\end{array}$ & $\begin{array}{l}48 \\
(23.2)\end{array}$ & $32(20.4)$ & - & - & $16(32.0)$ & - & - & - \\
\hline CTR, n (\%) & $\begin{array}{l}45 \\
(21.7)\end{array}$ & 35 (22.3) & 0 & - & $10(20.0)$ & 0 & - & - \\
\hline $\begin{array}{l}\text { Paravalvular } \\
\text { complications, } \\
\mathrm{n}(\%)\end{array}$ & $\begin{array}{l}61 \\
(29.5)\end{array}$ & $45(28.1)$ & 0 & - & $18(36.0)$ & 0 & - & - \\
\hline \multicolumn{9}{|c|}{$\begin{array}{l}\text { LVS, left-sided valve surgery only; TA, tricuspid annuloplasty; LVS + TA, left-sided valve surgery with } \\
\text { concomitant tricuspid annuloplasty; EF, ejection fraction; LA, left atrial; RA_LAD, right atrial long axis } \\
\text { diameter; RA_SAD, right atrial short axis diameter; LVEDD, left ventricular end-diastolic diameter; LVESD, } \\
\text { left ventricular end-systolic diameter; RV, right ventricular; IVS, interventricular septum; LVPW, left } \\
\text { ventricular posterior wall; PASP, pulmonary artery systolic pressure; TR, tricuspid regurgitation; MR, mitral } \\
\text { regurgitation; PR, pulmonary regurgitation; AR, aortic regurgitation; CTR, chordae tendineae rupture. }\end{array}$} \\
\hline \multicolumn{9}{|c|}{ * $\mathrm{P}<0.05$ for the echocardiographic changes $(\Delta)$ between LVS and LVS + TA group. } \\
\hline \multicolumn{9}{|c|}{ † $P<0.05$ versus LVS group preoperation. } \\
\hline \multicolumn{9}{|c|}{$\# \mathrm{P}<0.05$ postoperation versus preoperation. } \\
\hline
\end{tabular}

After surgical intervention, both the LVS group and the LVS + TA group manifested smaller cardiac chambers and alleviated valvular regurgitation. LVEF was decreased in both groups, indicating possible transient deterioration in cardiac function after surgery. However, the postoperative reductions of LA and RA diameter, MR and TR severity, as well as PASP were more significant in the LVS + TA group than in the LVS group $(\mathrm{P}<$ 0.05), implying the superiority of concomitant TA in improving echocardiagphic parameters.

\section{Complications and outcomes}

Stratified by surgical interventions with or without concomitant TA, the complications, surgical treatment and outcomes were summarized in Table 3. Of the 207 patients on admission, presentations of heart failure, systemic embolisms, neurological complications, and metastatic infection were 48 (23.2\%), 37 (17.9\%), 41 (19.8\%) and 26 (12.6\%) respectively. There was no evidence of significant differences in preoperative complications between the two treatment groups $(P>0.05)$. Of the 207 patients, a large proportion of patients underwent valve replacement instead of valvuloplasty $(97.6 \%$ vs. $2.4 \%)$. The LVS + TA group presented with significantly increased operation time ( $326 \pm 111$ vs. $278 \pm 95$ mins, $P=0.003)$ and postoperative complications rate compared with LVS group ( $44.0 \%$ vs. $23.6 \%, P=0.005)$. 
Table 3

Complications, Surgical treatment and Outcomes of the Patients with LSIE Stratified by Surgery Type.

\begin{tabular}{|lllll|}
\hline Variables & $\begin{array}{l}\text { All } \\
(\mathbf{n = 2 0 7 )}\end{array}$ & $\begin{array}{l}\text { LVS } \\
(\mathbf{n = 1 5 7 )}\end{array}$ & $\begin{array}{l}\text { LV + TA } \\
(\mathbf{n}=\mathbf{5 0})\end{array}$ & P value \\
\hline IE complications preoperation, $\mathrm{n}(\%)$ & & & & \\
\hline Heart failure & $48(23.2)$ & $35(22.3)$ & $13(26.0)$ & 0.589 \\
\hline Systemic embolism & $37(17.9)$ & $31(19.7)$ & $6(12.0)$ & 0.213 \\
\hline Neurologic complications & $41(19.8)$ & $31(19.7)$ & $10(20.0)$ & 0.969 \\
\hline Metastatic infection & $26(12.6)$ & $16(10.2)$ & $10(20.0)$ & 0.068 \\
\hline Left-sided valve surgery, $\mathrm{n}(\%)$ & & & & \\
\hline Valvuloplasty & $5(2.4)$ & $5(3.2)$ & 0 & 0.201 \\
\hline Valve replacement & $202(97.6)$ & $152(96.8)$ & $50(100)$ & \\
\hline Types of valve replaced, $\mathrm{n}(\%)$ & & & & \\
\hline Mechanical valve & $156(77.2)$ & $114(75.0)$ & $42(84.0)$ & 0.188 \\
\hline Biological valve & $46(22.8)$ & $38(25.0)$ & $8(16.0)$ & \\
\hline Postoperative complications, $\mathrm{n}(\%)$ & $59(28.5)$ & $37(23.6)$ & $22(44.0)$ & 0.005 \\
\hline Major Bleeding & $21(10.1)$ & $13(8.3)$ & $8(16.0)$ & 0.115 \\
\hline Neurological complications & $6(2.9)$ & $4(2.5)$ & $2(4.0)$ & 0.594 \\
\hline Cardiac complications & $24(11.6)$ & $15(9.6)$ & $9(18.0)$ & 0.104 \\
\hline Infection & $10(4.8)$ & $8(5.1)$ & $2(4.0)$ & 0.753 \\
\hline Thoracic effusion or pneumothorax & $7(3.4)$ & $5(3.2)$ & $2(4.0)$ & 0.781 \\
\hline Poor wound healing & $5(2.4)$ & $3(1.9)$ & $2(4.0)$ & 0.402 \\
\hline Operation time, min & $288 \pm 95$ & $278 \pm 88$ & $326 \pm 111$ & 0.003 \\
\hline Hospital stays (days) & $38 \pm 16$ & $38 \pm 16$ & $40 \pm 14$ & 0.359 \\
\hline Lost to follow up, $\mathrm{n}$ (\%) & $41(19.8)$ & $9(18.0)$ & $32(20.4)$ & 0.839 \\
\hline In-hospital mortality, $\mathrm{n}(\%)$ & $9(4.3)$ & $6(3.8)$ & $3(6.0)$ & 0.511 \\
\hline Long-term mortality after admission, $\mathrm{n}(\%)$ & $23(13.9)$ & $15(12.0)$ & $8(19.5)$ & 0.227 \\
\hline
\end{tabular}

LSIE, left-sided infective endocarditis; LVS, left-sided valve surgery only; LVS + TA, left-sided valve surgery with concomitant tricuspid annuloplasty.

*N $=166$ excluded 41 patients lost to follow up.

${ }^{\#} \mathrm{~N}=157$ excluded 41 patients lost to follow up and 9 patients died during hospitalization. 


\begin{tabular}{|c|c|c|c|c|}
\hline Variables & $\begin{array}{l}\text { All } \\
(n=207)\end{array}$ & $\begin{array}{l}\text { LVS } \\
(n=157)\end{array}$ & $\begin{array}{l}\text { LVS + TA } \\
(n=50)\end{array}$ & $P$ value \\
\hline Long-term mortality after discharge, $\mathrm{n}(\%) \#$ & $14(8.9)$ & $9(7.6)$ & $5(13.2)$ & 0.292 \\
\hline \multicolumn{5}{|c|}{$\begin{array}{l}\text { LSIE, left-sided infective endocarditis; LVS, left-sided valve surgery only; LVS + TA, left-sided valve surgery } \\
\text { with concomitant tricuspid annuloplasty. }\end{array}$} \\
\hline \multicolumn{5}{|l|}{ *N = 166 excluded 41 patients lost to follow up. } \\
\hline \multicolumn{5}{|c|}{${ }^{\#} \mathrm{~N}=157$ excluded 41 patients lost to follow up and 9 patients died during hospitalization. } \\
\hline
\end{tabular}

The average length of hospitalization was $38 \pm 16$ days and no significant differences were indicated in hospital stays between the two groups.

\section{Effect of concomitant TA on mortality}

Of the 207 patients, there were $9(4.3 \%)$ deaths during hospitalization. With a median follow-up duration of 34.4 (IQR 19.8-56.3) months, 14 (8.9\%) patients died after discharge (Table 3). A total of 41 (19.8\%) patients lost to follow up. However, demographic and baseline characteristic and complications data between followup and lost-to-follow-up were comparable (Supplementary Table 1-2). No significant differences were indicated in in-hospital mortality nor long-term mortality between the LVS group and the LVS + TA group. After being adjusted for Pitt bacteremia score and eGFR, concomitant TA was not significantly associated with inhospital mortality nor long-term mortality. However, Pitt bacteremia score was significantly associated with poor short-term and long-term prognosis $(P<0.001)$ (Table 4-5).

Table 4

Unadjusted and adjusted multivariable analyses of risk factors associated with mortality during index admission.

\begin{tabular}{|lllll|}
\hline Variables & Unadjusted & & Adjusted & \\
\cline { 2 - 5 } & OR $(95 \% \mathrm{Cl})$ & $\boldsymbol{P}$ value & OR $(95 \% \mathrm{Cl})$ & $P$ value \\
\hline Pitt score & $1.557(1.253-1.934)$ & $<0.001$ & $1.550(1.232-1.951)$ & $<0.001$ \\
\hline eGFR & $0.988(0.968-1.008)$ & 0.244 & $0.997(0.976-1.019)$ & 0.800 \\
\hline Concomitant TA & $1.606(0.387-6.673)$ & 0.514 & $1.757(0.323-9.570)$ & 0.515 \\
\hline OR, odds ratio; Cl, Confidence interval; TR, tricuspid regurgitation; TA, tricuspid annuloplasty. \\
\hline
\end{tabular}


Table 5

Unadjusted and adjusted multivariable analyses of risk factors associated with long-term mortality after index admission or after discharge.

\begin{tabular}{|lllll|}
\hline Variables & Unadjusted & \multicolumn{2}{l|}{ Adjusted } & \\
\cline { 2 - 5 } & HR $(95 \% \mathrm{Cl})$ & $\boldsymbol{P}$ value & HR (95\% Cl) & $P$ value \\
\hline Survival after index admission & & & & \\
\hline Pitt score & $1.535(1.317-1.790)$ & $<0.001$ & $1.508(1.281-1.75)$ & $<0.001$ \\
\hline eGFR & $0.983(0.970-0.996)$ & 0.010 & $0.995(0.983-1.008)$ & 0.474 \\
\hline Concomitant TA & $1.682(0.677-4.179)$ & 0.263 & $1.912(0.742-4.928)$ & 0.209 \\
\hline Survival after discharge & & & & \\
\hline Pitt score & $1.531(1.229-1.907)$ & $<0.001$ & $1.522(1.202-1.928)$ & $<0.001$ \\
\hline eGFR & $0.982(0.966-0.999)$ & 0.038 & $0.993(0.977-1.010)$ & 0.435 \\
\hline Concomitant TA & $2.300(0.745-7.099)$ & 0.148 & $2.693(0.830-8.741)$ & 0.099 \\
\hline
\end{tabular}

\section{Discussion}

As a common manifestation in LSIE, functional TR is thought to be associated with progressive right-sided heart failure and poor prognosis. The principle of concomitant TA at the time of LVS with certain indications was developed by virtue of other valvular diseases, including rheumatic heart disease and degenerative valvular heart disease [13]. However, there is a paucity of available evidence to elaborate on whether concomitant TA improves the prognosis of LSIE. Our study revealed that concomitant TA had significantly advanced effects on TR severity alleviation, right atrial dimension diminution, afterload reduction, as well as right heart function enhancement. However, concomitant TA at the time of LVS was associated with prolonged operation time, increased perioperative complications without improved short-term nor long-term prognosis.

In left-sided heart diseases, the right ventricular afterload usually increases (with or without pulmonary hypertension), followed by right ventricular remodeling and tricuspid annular dilation. Tricuspid annular dilation further results in poor leaflet apposition and leaflet coaptation mode, which eventually leads to functional TR [8]. The prevalence of functional TR with or without tricuspid annular dilatation in patients undergoing surgery for mitral regurgitation has ranged from 8-65\% [29-31]. Patients with higher functional TR was independently associated with worse prognosis [32]. According to previous study, concomitant TA at the time of LVS in patients with functional moderate to severe TR was associated with decreased TR degree and late TR progression, improved right ventricular function and remodeling, as well as better long-term prognosis without increasing surgical risks [33-39]. Left uncorrected at the time of LVS, mild or moderate degrees of functional TR may progress over time in approximately $25 \%$ of patients, and reoperation was associated with high mortality, resulting in reduced long-term survival $[13,40]$. Therefore, concomitant TA procedure for moderate to severe TR in left-sided surgery is a class I recommendation according to the American College of Cardiology/ American Heart Association and the European Society of Cardiology [13, 41]. 
However, according to Society of Thoracic Surgeons Adult Cardiac Surgery Database (STSACSD, version 2.73, 2011 to 2013), only 79\% of patients with severe TR and 39\% of patients with moderate TR undergo concomitant TA at the time of mitral surgery, possible due to the concern of safety and increased mortality or postoperative complications [42]. In our study, moderate to severe TR was presented in up to $24.8 \%$ of LSIE patients (Supplementary Table 3-4). Concomitant TA was performed in 50/207 (24.2\%) of patients undergoing LVS in our current study, including 22/143 (15.4\%) of patients with mild TR accompanied with tricuspid annulus dilation, and 24/45 (53.3\%) with moderate TR and 4/4 (100\%) of patients with severe TR. The TR severity was significantly improved after LVS independent of TA and the attenuation of TR in the LVS + TA group was significantly greater than that without TA, which was consistent with previous study [36]. The RA diameter and PASP were also significantly reduced after concomitant TA.

However, in our current study, concomitant TA at the time of LVS was associated with increased procedure time and postoperative complications without improved prognosis. According to previous study, operative mortality was almost double for multiple-valve procedures as compared with single-valve procedures. Moreover, patients with emergency status and endocarditis were significant associated with increased mortality in multiple-valve procedure [42]. Nearly one-quarter of IE patients with surgical indications do not undergo surgery, and operation for active IE was associated with high risk, with an overall in-hospital mortality of $20 \%[43,44]$. The possible reason was that patients with LSIE are usually urgent and progressive rapidly, which are quite different from rheumatic heart disease and degenerative heart disease with relatively latent progression and long duration. Therefore, whether functional tricuspid regurgitation should be treated at the time of LVS in patients with LSIE needs further robust clinical trials and evidence.

Another interesting finding in our study was that the risk of systemic embolisms was reduced in moderate to severe TR group (Supplementary Table 4), which was also elucidated among mitral stenosis patients in a previous study [45]. We speculate that the underlying rationale behind is the reduction of pulmonary arterial and venous flow with subsequent resolution of congestion and stasis in the left atrium [45].

\section{Strengths And Limitations}

To our knowledge, this is the first study evaluating the prognosis of concomitant TA in LSIE patients who underwent LVS. Nevertheless, this present study has several limitations. First, our study was a single-center retrospective study with quite small sample size, with inevitable selection bias to some extent. For instance, the proportion of patients with valve replacement in our study was remarkable higher than what the guideline recommends, possibly attributable to higher referral rate of surgery-suitable patients from regional hospitals and surgeon selection bias. Second, the diameter of tricuspid annulus was not routinely measured in our center over the study period, consequently we were not able to present and investigate the relationship between tricuspid annulus dilation and prognosis. Third, the rate of loss to follow-up was quite high in our study, however, the baseline characteristics were overall comparable. Although it's not easy to carry out a randomized control trial due to the relatively low incidence rate, further robust prospective multicenter randomized control trials with long-term follow-up are still necessary to explicate the prognostic value of concomitant TA with LVS in LSIE patients. 


\section{Conclusion}

Concomitant TA at the time of LVS significantly improved cardiac diameter but increased postoperative complications. It might not be associated with improved survival in LSIE patients.

\section{Declarations}

\section{Funding}

This study was funded by the National Natural Science Foundation of China (No. 81800345, No. 81770392, No. 81770394, No. 81800344, No. 82000372 and No. 82000385), Medical Research Foundation of Guangdong Province (No. A2018082) and China National Postdoctoral Program for Innovative Talents (No. BX20200400).

\section{Conflicts of interest}

The authors declare that they have no conflict of interest.

\section{Authors' contributions}

All the authors contributed to the design and development of the study, and reviewed the manuscript prior to submission. Yugang Dong and Chen Liu conceived and instructed the study. Zexuan Wu and Yuanyuan Zhou designed the study. Zexuan Wu wrote the original draft while Yili Chen and Fangfei Wei revised it. Zi Ye contributed to the English language editing. Xin He and Weihao Liang provided statistical expertise and supported the development of the statistical analysis plan. Jingjing Zhao, Ruicong Xue, Yuzhong Wu and Wengen Zhu supported the data collection as well as the follow up of the patients.

\section{Ethics approval}

All procedures performed in studies involving human participants were in accordance with the ethical standards of the institutional and/or national research committee and with the 1964 Helsinki declaration and its later amendments or comparable ethical standards.

\section{Informed Consent}

This study is a retrospective observational study with data collected from the electronic medical records of The First Affiliated Hospital of Sun Yat-Sen University. Informed consent was exempted in the study.

\section{Data Availability Statement}

All data used during the study are available from the corresponding author by request.

\section{References}

1. Wang A, Gaca JG, Chu VH. Management Considerations in Infective Endocarditis: A Review. JAMA. 2018;320:72-83. 
2. Habib G. Management of infective endocarditis. Heart. 2006;92:124-130.

3. Thuny F, Grisoli D, Collart F, Habib G, Raoult D. Management of infective endocarditis: challenges and perspectives. The Lancet. 2012;379:965-975.

4. San Roman JA, Vilacosta I, Lopez J, Sarria C. Critical Questions About Left-Sided Infective Endocarditis. J Am Coll Cardiol. 2015;66:1068-1076.

5. Baddour LM, Wilson WR, Bayer AS, et al. Infective Endocarditis in Adults: Diagnosis, Antimicrobial Therapy, and Management of Complications: A Scientific Statement for Healthcare Professionals From the American Heart Association. Circulation. 2015;132:1435-1486.

6. Kang DH, Lee S, Kim YJ, et al. Long-Term Results of Early Surgery versus Conventional Treatment for Infective Endocarditis Trial. Korean Circ J. 2016;46:846-850.

7. Kang DH, Kim YJ, Kim SH, et al. Early surgery versus conventional treatment for infective endocarditis. $\mathrm{N}$ Engl J Med. 2012;366:2466-2473.

8. Dreyfus GD, Martin RP, Chan KM, Dulguerov F, Alexandrescu C. Functional tricuspid regurgitation: a need to revise our understanding. J Am Coll Cardiol. 2015;65:2331-2336.

9. Nath J, Foster E, Heidenreich PA. Impact of tricuspid regurgitation on long-term survival. J Am Coll Cardiol. 2004;43:405-409.

10. Rogers JH, Bolling SF. The tricuspid valve: current perspective and evolving management of tricuspid regurgitation. Circulation. 2009;119:2718-2725.

11. Freed LA, Levy D, Levine RA, et al. Prevalence and clinical outcome of mitral-valve prolapse. N Engl J Med. 1999;341:1-7.

12. Pagnesi M, Montalto C, Mangieri A, et al. Tricuspid annuloplasty versus a conservative approach in patients with functional tricuspid regurgitation undergoing left-sided heart valve surgery: A study-level meta-analysis. Int J Cardiol. 2017;240:138-144.

13. Nishimura RA, Otto CM, Bonow RO, et al. 2014 AHA/ACC guideline for the management of patients with valvular heart disease: a report of the American College of Cardiology/American Heart Association Task Force on Practice Guidelines. J Am Coll Cardiol. 2014;63:e57-185.

14. Verdonk C, Darmon A, Cimadevilla C, et al. Is tricuspid annuloplasty increasing surgical mortality and morbidity during mitral valve replacement? A single-centre experience. Arch Cardiovasc Dis. 2018;111:480-486.

15. Li JS, Sexton DJ, Mick N, et al. Proposed modifications to the Duke criteria for the diagnosis of infective endocarditis. Clin Infect Dis. 2000;30:633-638.

16. Levey AS, Stevens La Fau - Schmid CH, Schmid Ch Fau - Zhang YL, et al. A new equation to estimate glomerular filtration rate.

17. Ho KK, Pinsky JL, Kannel WB, Levy D. The epidemiology of heart failure: the Framingham Study. J Am Coll Cardiol. 1993;22:6A-13A.

18. King M, Kingery J, Casey B. Diagnosis and evaluation of heart failure. Am Fam Physician. 2012;85:11611168.

19. Chan KL, Dumesnil Jg Fau - Cujec B, Cujec B Fau - Sanfilippo AJ, et al. A randomized trial of aspirin on the risk of embolic events in patients with infective endocarditis. 
20. García-Cabrera E, Fernández-Hidalgo N, Almirante B, et al. Neurological complications of infective endocarditis: risk factors, outcome, and impact of cardiac surgery: a multicenter observational study. Circulation. 2013;127:2272-2284.

21. Revilla A, Lopez J, Vilacosta I, et al. Clinical and prognostic profile of patients with infective endocarditis who need urgent surgery. Eur Heart J. 2007;28:65-71.

22. Habib G, Lancellotti P, Antunes MJ, et al. 2015 ESC Guidelines for the management of infective endocarditis: The Task Force for the Management of Infective Endocarditis of the European Society of Cardiology (ESC). Endorsed by: European Association for Cardio-Thoracic Surgery (EACTS), the European Association of Nuclear Medicine (EANM). Eur Heart J. 2015;36:3075-3128.

23. Feldman C, Alanee S, Yu VL, et al. Severity of illness scoring systems in patients with bacteraemic pneumococcal pneumonia: implications for the intensive care unit care. Clin Microbiol Infect. 2009;15:850-857.

24. Zoghbi W. Recommendations for evaluation of the severity of native valvular regurgitation with twodimensional and doppler echocardiography. Journal of the American Society of Echocardiography. 2003; 16:777-802.

25. Yock PG, Popp RL. Noninvasive estimation of right ventricular systolic pressure by Doppler ultrasound in patients with tricuspid regurgitation. Circulation. 1984 Oct;70(4):657-62.

26. Jeong DS, Kim KH. Tricuspid annuloplasty using the MC3 ring for functional tricuspid regurgitation. Circ J. 2010;74:278-283.

27. Hwang HY, Chang HW, Jeong DS, Ahn H. De Vega annuloplasty for functional tricupsid regurgitation: concept of tricuspid valve orifice index to optimize tricuspid valve annular reduction. J Korean Med Sci. 2013;28:1756-1761.

28. Sohn SH, Kim KH, Lee Y, Choi JW, Hwang HY. Long-term outcomes of rigid ring versus De Vega annuloplasty for functional tricuspid regurgitation: A propensity score-matching analysis. J Thorac Cardiovasc Surg. 2019.

29. Dreyfus GD, Corbi PJ, Chan KMJ, Bahrami T. Secondary tricuspid regurgitation or dilatation: which should be the criteria for surgical repair? Ann Thorac Surg. 2005;79:127-132.

30. Chikwe J, Itagaki S, Anyanwu A, Adams DH. Impact of Concomitant Tricuspid Annuloplasty on Tricuspid Regurgitation, Right Ventricular Function, and Pulmonary Artery Hypertension After Repair of Mitral Valve Prolapse. J Am Coll Cardiol. 2015;65:1931-1938.

31. Chan V, Burwash IG, Lam BK, et al. Clinical and echocardiographic impact of functional tricuspid regurgitation repair at the time of mitral valve replacement. Ann Thorac Surg. 2009;88:1209-1215.

32. Benfari G, Antoine C, Miller WL, et al. Excess Mortality Associated With Functional Tricuspid Regurgitation Complicating Heart Failure With Reduced Ejection Fraction. Circulation. 2019;140:196-206.

33. Van de Veire NR, Braun J, Delgado V, et al. Tricuspid annuloplasty prevents right ventricular dilatation and progression of tricuspid regurgitation in patients with tricuspid annular dilatation undergoing mitral valve repair. J Thorac Cardiovasc Surg. 2011;141:1431-1439.

34. Lee H, Sung K, Kim WS, et al. Clinical and hemodynamic influences of prophylactic tricuspid annuloplasty in mechanical mitral valve replacement. J Thorac Cardiovasc Surg. 2016;151:788-795. 
35. Sakata T, Mogi K, Sakurai M, et al. Effect of tricuspid annuloplasty concomitant with left heart surgery on right heart geometry and function. J Thorac Cardiovasc Surg. 2018;156:1050-1061.

36. Desai RR, Vargas Abello LM, Klein AL, et al. Tricuspid regurgitation and right ventricular function after mitral valve surgery with or without concomitant tricuspid valve procedure. J Thorac Cardiovasc Surg. 2013;146:1126-1132 e1110.

37. Ye Y, Desai R, Vargas Abello LM, et al. Effects of right ventricular morphology and function on outcomes of patients with degenerative mitral valve disease. J Thorac Cardiovasc Surg. 2014;148:2012-2020 e2018.

38. Pingpoh C, Nuss S, Kueri S, et al. Adding tricuspid repair to standard open heart surgery does not increase risk but improves right ventricular function. Interact Cardiovasc Thorac Surg. 2019;29:416-421.

39. Badhwar V, Rankin JS, He M, et al. Performing Concomitant Tricuspid Valve Repair at the Time of Mitral Valve Operations Is Not Associated With Increased Operative Mortality. Ann Thorac Surg. 2017;103:587593.

40. Jeong DS, Park PW, Mwambu TP, et al. Tricuspid reoperation after left-sided rheumatic valve operations. Ann Thorac Surg. 2013;95:2007-2013.

41. Baumgartner H, Falk V, Bax JJ, et al. 2017 ESC/EACTS Guidelines for the management of valvular heart disease. Eur Heart J. 2017;38:2739-2791.

42. Vassileva CM, Li S, Thourani VH, et al. Outcome characteristics of multiple-valve surgery: comparison with single-valve procedures. Innovations (Phila). 2014;9:27-32.

43. Chu VH, Park LP, Athan E, et al. Association between surgical indications, operative risk, and clinical outcome in infective endocarditis: a prospective study from the International Collaboration on Endocarditis. Circulation. 2015;131:131-140.

44. Cahill TJ, Baddour LM, Habib G, et al. Challenges in Infective Endocarditis. J Am Coll Cardiol. 2017;69:325-344.

45. Chiang CW, Lo SK, Kuo CT, Cheng NJ, Hsu TS. Noninvasive predictors of systemic embolism in mitral stenosis. An echocardiographic and clinical study of 500 patients. Chest. 1994;106:396-399.

\section{Figures}




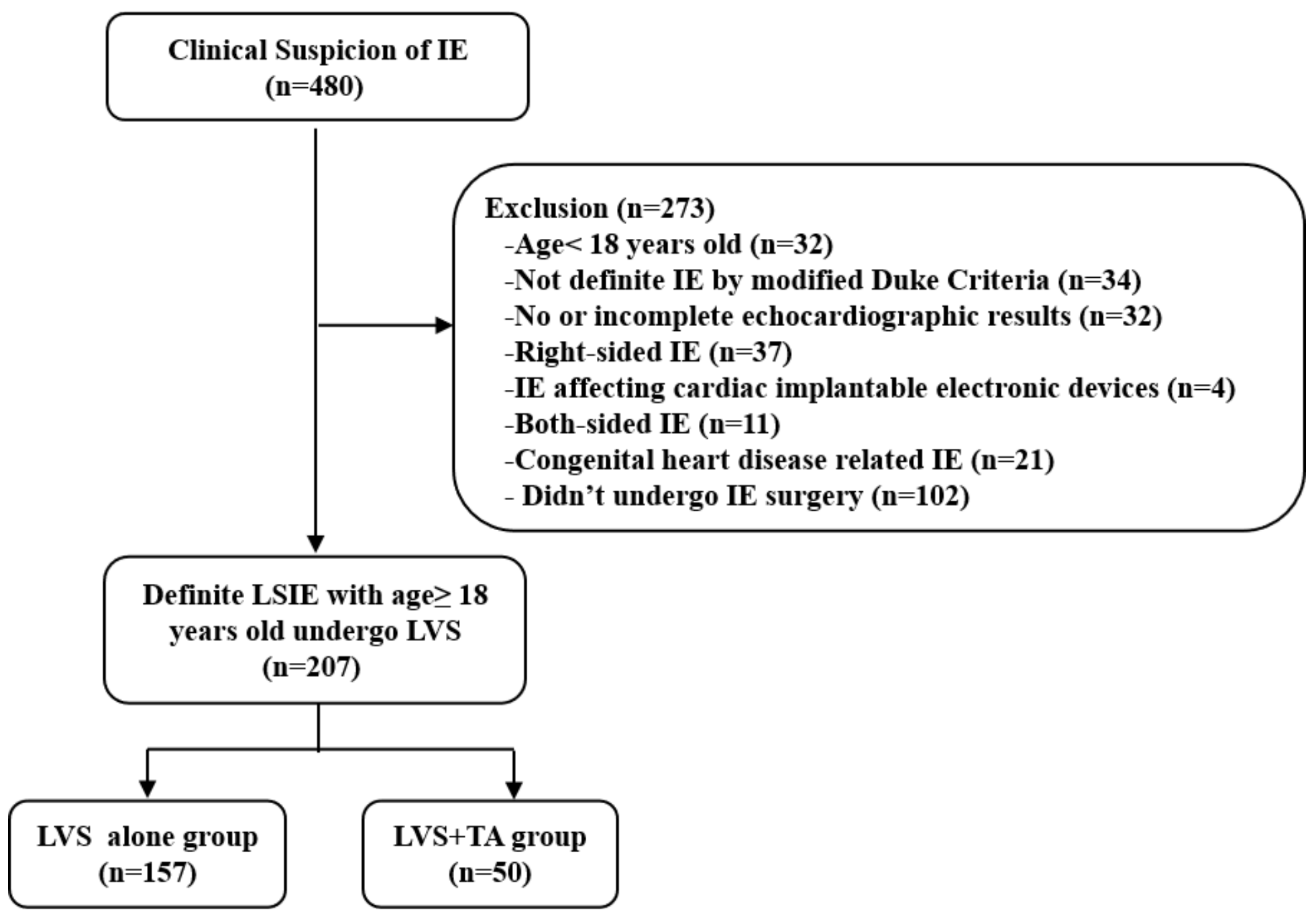

Figure 1

Flowchart of the study. IE, infective endocarditis; LSIE, left-sided infective endocarditis; LVS, left-sided valve surgery only; LVS+ TA, left-sided valve surgery with concomitant tricuspid annuloplasty.

\section{Supplementary Files}

This is a list of supplementary files associated with this preprint. Click to download.

- Supplementarytables.docx 